\title{
Géneros pedagógicos en evaluaciones escritas de Biología e Historia en la Enseñanza Media*
}

Pedagogical Genres in Written Evaluations of Biology and History in Secondary Education

Gêneros pedagógicos em avaliações escritas de Biologia e História no Ensino Médio

Valeska Müller** iD orcid.org/0000-0002-5484-698X

Para citar este artículo: Müller, V. (2O21). Géneros pedagógicos en evaluaciones escritas de Biología e Historia en la Enseñanza Media. Revista Colombiana de Educación, /(82), 107-130. https://doi.org/10.17227/ rce.num82-10470

\begin{tabular}{lr}
\hline (C) $\$$ BY & Recibido: $01 / 09 / 2019$ \\
Evaluado: $07 / 09 / 2020$
\end{tabular}

* $\quad$ Este artículo es parte de una investigación más amplia realizada en una tesis doctoral que contó, en su fase de redacción final, con el apoyo del Proyecto Fondecyt Regular n. 1130474.

** Universidad de Los Lagos, Chile, Departamento de Humanidades y Arte. Correo: valeska.muller@ulagos.cl 


\section{Resumen}

Este artículo de investigación presenta un análisis comparativo de los géneros pedagógicos de las áreas de Biología e Historia, producidos por estudiantes de dos niveles de enseñanza media ( $1^{\circ}$ y $3^{\circ}$ medio) en evaluaciones escritas. El concepto de género y la tipología en que se basa este estudio se fundamenta en el desarrollo sobre alfabetización impulsado por la Escuela de Sidney (Rose y Martin, 2012; Martin y Rose, 2008). Los resultados del análisis de las preguntas y respuestas obtenidas de 20 evaluaciones estudiantiles muestran que en el área de Biología el género del reporte está presente en ambos niveles, así como las explicaciones secuenciales y factoriales, mientras que en el área de Historia los géneros pedagógicos se concentran en el relato histórico y en las explicaciones, que colaboran con la comprensión de procesos y que incorporan, ligeramente, la expresión de juicios y puntos de vista. Las diferencias en los géneros utilizados por los estudiantes reflejan de manera incipiente las distinciones entre estructuras del conocimiento científico y humanístico

\section{Palabras clave}

Lenguaje académico; escritura; literacidad; evaluación estudiantil; investigación en escritura

\section{Keywords}

Academic language; writing;

literacy; student evaluation; writing research

\begin{abstract}
This research article shows a comparative analysis of pedagogical genres in the Biology and History areas, produced by two levels of high school students (lst and 3rd grade) in written evaluations. The concept of gender and the typology used in this study are based on the literacy developments promoted by the School of Sydney (Rose y Martin, 2012, Martin y Rose, 2008). The results of the analysis of the questions and answers obtained from 20 student evaluations, show that in the area of Biology the gender of the report is present in both levels, as well as the sequential and factorial explanations. In the area of History, the pedagogical genres concentrate on the historical narrative and explanations, which collaborate with the understanding of the processes and that, slightly, incorporate expression of judgments and various points of view. The differences in the genres used by the students, reflect the distinctions between structures of scientific and humanistic knowledge incipiently.
\end{abstract}

\section{Resumo}

Este artigo de pesquisa apresenta uma análise comparativa dos gêneros pedagógicos das áreas de Biologia e História, produzidos por alunos de dois níveis do Ensino Médio $\left(1^{\circ}\right.$ e $3^{\circ}$ ano) em avaliações escritas. O conceito de gênero e a tipologia em que este estudo se baseia tem como base o desenvolvimento da alfabetização promovido pela Escola de Sydney (Rose e Martin, 2012; Martin e Rose, 2008). Os resultados da análise das questões e respostas obtidas nas avaliações de 20 alunos mostram que na área da Biologia o gênero do relatório está presente em ambos os níveis, bem como nas explicações sequenciais e fatoriais, enquanto na área da História os gêneros pedagógicos centram-se no relato e nas explicações históricas, que ajudam a compreender os processos e que pouco incorporam a expressão de julgamentos e pontos de vista. As diferenças nos gêneros utilizados pelos alunos estão refletindo incipientemente as distinções entre as estruturas do conhecimento científico e humanístico.

\section{Palavras-chave}

Linguagem acadêmica; escritura; literacia; avaliação estudiantil; pesquisa em escritura 


\section{Introducción}

En el desarrollo de una didáctica de la escritura, las contribuciones realizadas por la lingüística sistémico-funcional, la teoría del registro y el género y la Escuela de Sidney ${ }^{1}$ han puesto el foco en el concepto de género. Martin y Rothery (1993) señalan que, para introducir a los maestros y profesores en un modelo funcional del lenguaje, es preciso comenzar con dicho concepto, es decir, con los diferentes tipos de texto usados en nuestra cultura para hacer cosas. La importancia del género en la enseñanza de la escritura ha sido destacada por numerosos investigadores y docentes, entre ellos Christie (2012), para quien el análisis y la consideración de un género en relación con sus significados, patrones lingüísticos y estructura global no solo permiten enseñar a escribir, sino también introducir los significados para lograr una comprensión más profunda de un conocimiento.

Desde la perspectiva de la lingüística sistémico-funcional, el conocimiento y su expresión están fuertemente entrelazados, por lo que el desarrollo de uno conlleva el del otro. En este sentido, apropiarse de una forma de construir el conocimiento implica incorporar un modo de pensar. Es por ello, y por el papel que desempeña el contexto en la determinación de los modos de expresión, que las investigaciones desarrolladas desde esta perspectiva destacan las diferencias entre el uso de la lengua en contextos académicos y en contextos familiares o de transmisión de significados del sentido común, y, en el contexto académico, entre el uso de la lengua en las ciencias y en las humanidades.

Los estudios sobre alfabetización en contextos académicos han dejado de manifiesto que los modos de leer y escribir no son iguales en todos los ámbitos ni en todas las disciplinas y que, por tanto, existen culturas diferentes en torno a lo escrito. Esta afirmación, válida para el conocimiento especializado, debiera manifestarse también en instancias de formación menos especializadas, como es el caso de la Educación Media, y tras esta búsqueda es pertinente analizar los géneros discursivos pedagógicos que producen los estudiantes en dos campos del conocimiento: la biología y la historia.

En consecuencia, el objetivo de este estudio es analizar comparativamente los géneros pedagógicos producidos por estudiantes chilenos de enseñanza secundaria en las disciplinas de biología e historia en contextos de evaluación escrita. Este acercamiento a la escritura estudiantil a propósito de la evaluación implica comprender los textos (pruebas o test) no solo como preguntas y respuestas, sino como recontextualizaciones del

1 El término Escuela de Sidney fue acuñado en 1994 para hacer referencia a los trabajos en lingüística y educación que James Martin lideraba desde los años ochenta en el Departamento de Lingüística de la Universidad de Sidney. 
conocimiento que cumplen un propósito global y configuran un género que tiene que ver tanto con las exigencias que plantea el docente como con el modo como el estudiante reconstruye el conocimiento acerca del cual se indaga. Por otra parte, las disciplinas escogidas responden al interés por detectar si estas prácticas de recontextualización manifiestan en alguna medida las particularidades del discurso científico y de las ciencias sociales y la historia, estudiadas por Halliday y Martin (1993).

Halliday (1993), en consonancia con Bernstein (1999), sugiere que no es posible hacer ciencia usando el lenguaje de todos los días, que el lenguaje de las ciencias ha evolucionado en cierta forma, por los significados que se han creado en el discurso científico y por la necesidad de nombrar los principios generales que se derivan de los experimentos. A partir de esta idea, en el marco de la lingüística sistémico-funcional, numerosos autores se han ocupado de contrastar el discurso de las ciencias y el de las humanidades (Eggins et ál., 1993; Halliday y Martin, 1993, Martin et ál., 1988 y Wignell, 2007), y han identificado el discurso histórico como más típico de las humanidades. Esto significaría que su verticalidad es débil, ya que el conocimiento estaría organizado de manera segmentada (tema por tema o periodo por periodo) sin demasiados elementos técnicos interconectados en una superestructura conceptual.

Considerando lo expuesto hasta este punto, el interés de esta investigación radica no solo en la posibilidad de analizar la escritura estudiantil desde los presupuestos teóricos de la lingüística sistémico-funcional y la teoría del registro y el género para detectar qué géneros están presentes y cómo se constituyen, sino también en visualizar de qué manera la ciencia y la historia se manifiestan en la escritura en el nivel de estudiantes de educación secundaria.

\section{Constructo teórico-analítico}

Los trabajos sobre alfabetización desde las perspectivas de la lingüística sistémico-funcional y la teoría del registro y el género comenzaron en la década de los 80. Estos nuevos planteamientos pusieron en entredicho la idea de que la capacidad para leer y escribir es una competencia o un estado básico que se logra de una vez y para siempre, y dieron a entender que la alfabetización es un proceso, un saber que se desarrolla en contacto con los distintos contextos y, por ello, varía y debe ser objeto de atención de la didáctica de la lengua en todo momento de la formación de un estudiante (Christie, 1998; Halliday y Martin, 1993; Martin, 2000; Martin y Rothery, 1993). 


\section{La concepción sociosemiótica de la lengua y las variables del contexto}

En el marco de la lingüística sistémico-funcional, el lenguaje se entiende como un sistema semiótico que realiza y manifiesta las estructuras semióticas que conforman una sociedad y una cultura, por lo que lenguaje y contexto se determinan bidireccionalmente (Halliday y Hasan, 1989; Halliday y Martin, 1993). Del mismo modo que el lenguaje tiene tres funciones generales (o metafunciones), el contexto social de uso del lenguaje puede examinarse desde estas tres perspectivas. De tal manera, en relación con la metafunción interpersonal, el contexto social es visto como tenor de las relaciones sociales (quiénes están participando y qué relación existe entre ellos); en relación con la metafunción ideacional, el contexto social se presenta como campo de la experiencia (acerca de qué se habla o qué está ocurriendo) y en relación con la metafunción textual, el contexto es visto como modo de comunicación (cómo funciona el lenguaje o la organización simbólica del texto) (Eggins y Martin, 2003)

A continuación, la interpretación que hace Martin de la relación entre lenguaje y contexto social implica reconocer el contexto como el estrato más alto del significado, que posee un plano contextual superior -la ideología - que hace posible incorporar al modelo una interpretación crítica de los planos comunicativos inferiores, además de permitir la configuración de sistemas de géneros, en atención a los contextos locales y culturales en que circulan (Martin, 2014). El desarrollo de estos planteamientos ha permitido Ilevar a cabo análisis que integran la noción de género, desarrollada por la teoría del registro y el género y la Escuela de Sidney, y la de registro, desarrollada por la lingüística sistémico-funcional (Rose y Martin, 2012; Morris y Navarro, 2011).

\section{El género como actividad social que se realiza en los textos}

Dentro del contexto semióticamente estratificado, en un nivel de mayor abstracción, se encuentra el propósito social global de un texto, es decir, el género (Rose y Martin, 2012). Este es responsable de legitimar las combinaciones de tenor (relaciones), campo (actividad) y modo (rol del lenguaje) en una cultura (Martin, 1985; Moyano, 2013a); y de "mostrar cómo el propósito social perseguido se logra a través de una serie de pasos que constituyen su estructura" (Moyano, 2013a, p. 46). En este sentido, el género se organiza en estructuras esquemáticas dado que es esperable que a un paso siga otro, construyendo así patrones de pasos o etapas que dan cuenta de una estrategia para lograr el propósito hacia el cual se dirige. Ahora bien, un texto puede tener múltiples propósitos; sin embargo, es el primario el que modela sus pasos y establece la familia de géneros a la cual pertenece. 
Martin (1992) y Martin y Rose (2008) definen género como una actividad social orientada a una meta, organizada en pasos o etapas y en la cual los hablantes se interrelacionan como miembros de su cultura. El modelo funcional del lenguaje muestra cómo los géneros alcanzan típicamente sus metas (objetivos o propósitos) mediante la organización del texto en pasos o etapas. La identificación de los pasos de un texto con una función particular es consecuencia de la selección de patrones de lenguaje en cada uno de ellos. Por ende, analizar un texto permitirá determinar su estructura esquemática o genérica e inferir el tipo de actividad social, el propósito perseguido y el modo como se lleva a cabo (Moyano, 2013a).

En la propuesta de Martin y Rothery (1993), este modo de observar los textos es importante para enseñar a los estudiantes la escritura de diferentes géneros. Conocer las etapas de un género, poder identificarlas, saber cómo se usa el lenguaje para significar en diferentes géneros y cómo estos funcionan en un contexto social y académico determinado es importante para ayudar a los estudiantes en su escritura. Lo anterior implica explicitar ante los alumnos el modo como el uso de la lengua "construye significado y cómo lo hace en los diferentes géneros, prestando especial interés al contenido, la estructura de los textos y los patrones gramaticales para la realización del registro" (Moyano, 2007, sección 2, párrafo 1).

Si bien la propuesta de Martin y Rose (2008) en el ámbito de los géneros es amplia y permite el análisis de varios de ellos, el interés particular de la Escuela de Sidney por trabajar con textos producidos por estudiantes de la escuela primaria y secundaria ha permitido acuñar el término "géneros pedagógicos"2 (Rose y Martin, 2012) y diseñar un mapa de los géneros escolares, que organiza y sintetiza aquellos que habitualmente producen los estudiantes como parte de sus tareas educativas. Dicho mapa o esquema recoge lo esencial de otros esquemas presentados para el contexto escolar en Historia (Coffin, 2000) y en Ciencia (Veel, 2000) y ha sido el resultado de una larga investigación acerca de los géneros que los estudiantes australianos necesitan manejar para tener éxito en la escuela secundaria. En esta investigación, este mapa ha sido útil para identificar de los géneros que se utilizan en el nivel de enseñanza media en Chile, dado que se trata de géneros básicos, ligados a habilidades cognitivas que se pretende desarrollar en los estudiantes. De hecho, los nombres para cada género (entretener-comprometer, informar y evaluar ${ }^{3}$ ) constituyen, según sugieren Rose y Martin (2012), el primer nivel de metalenguaje que los profesores pueden usar para enseñar a leer y escribir.

2 Rose y Martin (2012) utilizan el término géneros pedagógicos para referirse a la "pedagogía basada en el género", concepto con el que aludían a la enseñanza de estrategias diseñadas para guiar a los estudiantes a escribir los géneros de la escuela.

3 En inglés: engaging, informing y evaluating (Rose y Martin, 2012). 
Los pasos o etapas que se llevan a cabo para lograr el propósito perseguido se realizan en el texto como una estructura que indica que los contenidos se distribuyen a medida que el texto se despliega (Moyano, 2013a). Dicha estructura se determina describiendo la composición estructural o estructura de constituyentes del texto, es decir, reconociendo las diferentes partes que componen la interacción completa. Como indica Eggins (1994), una vez que comenzamos a dividir un texto en sus constituyentes, debemos considerar sobre qué base estableceremos que dos partes de un texto conforman pasos separados. Existen, esencialmente, dos clases de criterios que podemos usar: un criterio formal que divide un texto en pasos/partes de acuerdo con la forma de los constituyentes, y un criterio funcional que divide el género en pasos/partes según la función de los constituyentes. A continuación, se hace necesario explicar cómo se relacionan las partes entre sí para componer el todo. Este objetivo puede alcanzarse mediante un etiquetamiento funcional en la descripción genérica. La etiqueta debe describir qué hace una etapa, en relación con el todo, en los términos más específicos que puedan ser establecidos.

En el análisis del género, Eggins (1994) recomienda evitar las etiquetas vacías de contenido, tales como comienzo, medio, fin, o introducción, desarrollo, conclusión, dado que no son específicas de un género (todos ellos tienen comienzo, medio y fin). Es necesario encontrar etiquetas apropiadas; para ello, hay que preguntarse, por ejemplo, qué es exactamente lo que se hace en este comienzo del texto o qué se hace en el cuerpo de un ensayo, que es diferente de lo que se hace en el cuerpo de otro género. También es posible descubrir cuáles son elementos obligatorios, definitorios del género, preguntándose qué pasos podrían ser eliminados sin que el texto deje de ser el que es.

En el caso del mapa de los géneros de la escuela identificados por Rose y Martin (2012), los autores proponen también una denominación para las etapas de cada género, esto es, una estructura genérica, y señalan que este es el segundo nivel de metalenguaje que los profesores deben conocer.

De acuerdo con Eggins (1994), aunque identificar la estructura de un género es lo fundamental para su análisis, este no puede llevarse a cabo sin examinar las realizaciones de cada uno de sus elementos. En efecto, relacionar los elementos de la estructura con sus realizaciones lingüísticas es el procedimiento central del análisis genérico, puesto que permite reconocer los patrones de realización propios de cada género, e incluso más, los de cada paso de la estructura de cada género.

En el reconocimiento de los patrones de realización ya mencionados intervienen seis sistemas de recursos, que han sido descritos por Martin y Rose (2008) y utilizados en español por Moyano (2013a). Se trata de la ideación y conjunción para el significado ideacional; valoración y negociación para el 
significado interpersonal, e identificación y periodicidad para el significado textual. Aquí nos referiremos a los dos primeros, puesto que en ellos se centrará el posterior análisis.

La ideación tiene que ver con la naturaleza del conocimiento que se despliega en el texto, ya sea este cotidiano, especializado o académico. Se centra en las relaciones entre procesos, participantes y circunstancias. La conjunción da cuenta de los significados ideacionales lógicos que establecen relaciones de distinto tipo entre los procesos presentes en el texto. También construye las relaciones lógico-semánticas (de taxis) en el texto.

\section{El discurso de la ciencia, las ciencias sociales y las humanidades}

La investigación en esta área de estudio ha explorado las diferencias y conexiones entre el discurso de la ciencia, de las ciencias sociales y las humanidades. Ahora bien, sin negar la importancia de la abstracción en el discurso de las humanidades y de la historia, es preciso decir que ninguno de ellos se encuentra exento de tecnicismos, aunque estos puedan ser diferentes en uno y otro. Quizá la mayor diferenciación entre el discurso científico y el histórico se halla — como el propio Martin señala - en el hecho de que mientras que la ciencia se relaciona con la construcción de taxonomías y la implicación de secuencias acerca de cómo es el mundo, la historia se relaciona con la construcción del texto como documento acerca de lo que sucede en el mundo, y para ello generaliza (Martin, 1993a). En efecto, en la historia "no se trata de presentar individuos llevando a cabo acciones particulares, sino [...] grupos de personas, tratados como clases genéricas [...], participando de clases genéricas de eventos" (Moyano, 2013a, p. 67). Asimismo, la nominalización en ciencia está fuertemente asociada con las definiciones; su función es acumular significados por medio de términos técnicos. En historia, por su parte, la nominalización está fuertemente asociada con la realización de eventos como participantes, así como con las realizaciones lógicas que tienen lugar dentro de la cláusula, al tiempo que permite la estructuración de la información en un texto (Martin, 1993a).

\section{Metodología}

\section{Corpus}

En esta investigación de carácter exploratorio, que busca analizar comparativamente los géneros pedagógicos presentes en evaluaciones escritas, se trabaja sobre la base de 20 pruebas de desarrollo de las áreas de Biología 
e Historia, producidas por estudiantes chilenos de primer y tercer año de Educación Media, provenientes de un liceo municipalizado mixto de excelencia académica.

La selección de este liceo responde a que se trata de una muestra dirigida hacia estudiantes que, en su mayoría, aspiran a proseguir sus estudios en la educación superior universitaria, puesto que este interés indicaría una mayor preocupación por lograr un mejor desarrollo de las capacidades para leer y escribir textos en registros académicos. Las áreas seleccionadas -Biología e Historia - se relacionan con la propuesta de géneros diferentes en el campo de las ciencias y las humanidades.

En relación con la producción escrita, se ha optado por el análisis de textos elaborados en contextos reales de evaluación, ya que la escritura individual manuscrita permite apreciar de manera directa el manejo que los estudiantes poseen de los recursos lingüísticos para la construcción de significados en diferentes disciplinas, desde el punto de vista funcional.

\section{Procedimiento para el análisis de los textos estudiantiles}

El análisis sigue las perspectivas metodológicas que aportan la gramática sistémico-funcional y la teoría del registro y el género, a la vez que implican abordar los diferentes niveles estratificados del contexto (género y registro) y del lenguaje (nivel discursivo-semántico y nivel léxico-gramatical). La caracterización de un género implica definir el contexto cultural en el que se lleva a cabo la actividad social, el tipo de situación o registro (tenor, campo y modo), el propósito y los pasos para lograrlo, lo que revela su estructura. Por otra parte, se hace necesario analizar el uso del lenguaje en el estrato discursivo semántico, con referencias al estrato léxico-gramatical (Moyano, 2013b). En consecuencia, el abordaje analítico de los textos estudiantiles se llevó a cabo en dos etapas: en la primera, el estudio se centra en las variables registrales y se procede a identificar las características generales del tipo de situación social correspondiente a los textos estudiantiles. En la determinación del tenor de un texto se explicitan las relaciones interpersonales entre interlocutores. En este caso, el docente propone al estudiante una serie de preguntas que este responderá de acuerdo con sus conocimientos y con el tiempo con el que cuenta para desarrollar la tarea. Para la determinación del campo se consideran los ítemes lexicales presentes en los textos (Eggins, 1994). En este caso, a partir del tema de las preguntas que los docentes dirigen a sus estudiantes, es posible precisar de qué tratan (o debieran tratar) los textos. En el cuadro 1 se presentan las consignas que los profesores entregan a sus estudiantes. 


\section{Cuadro 1.}

Consignas de los profesores según niveles y áreas de estudio

\begin{tabular}{|c|c|c|c|}
\hline Área & Preguntas & $1 .^{\circ}$ medio & 3. ${ }^{\circ}$ medio \\
\hline \multirow{6}{*}{ Biología } & $\mathrm{Pl}$ & $\begin{array}{l}\text { Describe los tres principales } \\
\text { factores que determinan la } \\
\text { pérdida de energía en las } \\
\text { interacciones tróficas. }\end{array}$ & $\begin{array}{l}\text { El topo es un mamífero } \\
\text { excavador. Describa cómo } \\
\text { explicaría Lamarck el hecho de } \\
\text { que tenga ojos tan pequeños. }\end{array}$ \\
\hline & p2 & $\begin{array}{l}\text { De acuerdo con sus } \\
\text { características, ¿qué tipo } \\
\text { de pirámide se representa } \\
\text { en la ilustración? }\end{array}$ & $\begin{array}{l}\text { (A propósito de un experimentc } \\
\text { con aves) ¿Qué aves } \\
\text { atrajeron un menor y mayor } \\
\text { número de hembras? }\end{array}$ \\
\hline & P3 & $\begin{array}{l}\text { Señala qué seres vivos } \\
\text { podrían ser parte de } \\
\text { esta pirámide trófica. }\end{array}$ & $\begin{array}{l}\text { Elabora una conclusión } \\
\text { mencionando cuál es el } \\
\text { factor selectivo y su relación } \\
\text { con los tipos de selección. }\end{array}$ \\
\hline & p4 & $\begin{array}{l}\text { Analiza el siguiente texto } \\
\text { y gráfico y responde, ¿en } \\
\text { cuál de los dos periodos } \\
\text { de estudio la productividad } \\
\text { fue mayor? ¿Qué factores } \\
\text { aumentaron la productividad? }\end{array}$ & $\begin{array}{l}\text { La siguiente figura muestra un } \\
\text { registro fósil recién encontrado. } \\
\text { Antes de empezar cualquier } \\
\text { estudio, ¿qué se puede decir } \\
\text { sobre la naturaleza de los } \\
\text { individuos? ¿Se habrán hecho } \\
\text { al mismo tiempo las huellas? }\end{array}$ \\
\hline & p5 & $\begin{array}{l}\text { ¿De qué manera se relacionan } \\
\text { las interacciones tróficas } \\
\text { con el aumento de la } \\
\text { productividad de fitoplancton? }\end{array}$ & $\begin{array}{l}\text { Elabora una hipótesis } \\
\text { que explique la serie de } \\
\text { eventos representados. }\end{array}$ \\
\hline & p6 & $\begin{array}{l}\text { Establece dos conclusiones a } \\
\text { partir de la información anterior. }\end{array}$ & $\begin{array}{l}\text { ¿Qué habrá demostrado el } \\
\text { experimento de Weismann? } \\
\text { ¿Apoyan su resultado las } \\
\text { ideas de Lamarck? }\end{array}$ \\
\hline \multirow{3}{*}{ Historia } & $\mathrm{Pl}$ & $\begin{array}{l}\text { Explique la evolución } \\
\text { del mundo rural. }\end{array}$ & $\begin{array}{l}\text { Explique por qué se plantea } \\
\text { que a partir del gobierno } \\
\text { militar se habría suprimido } \\
\text { el Estado de Derecho }\end{array}$ \\
\hline & p2 & - & $\begin{array}{l}\text { Caracterice la doctrina de } \\
\text { la seguridad nacional }\end{array}$ \\
\hline & P3 & - & $\begin{array}{l}\text { Explique en qué consistió } \\
\text { el proyecto de refundación } \\
\text { política del régimen militar }\end{array}$ \\
\hline
\end{tabular}

Fuente: elaboración propia

En la determinación del modo, que se refiere al canal de comunicación y a las formas en que se estructuran los textos, es central considerar el tipo de preguntas (abiertas o cerradas) y la organización que adoptan las respuestas de los estudiantes a las consignas, además del capital verbal (cantidad de palabras) en uso. 
En la segunda etapa, se desarrolla el estudio del género, con énfasis en la variable campo y en los patrones discursivo-semánticos y léxico-gramaticales de la ideación y la conjunción (Martin y Rose, 2008; Moyano, 2013b). Para ello, se precisaron los propósitos a los que se dirigían los textos que investigamos y las etapas que los conformaban. Cada pregunta-respuesta fue considerada una unidad con un propósito definido, en primera instancia, en la instrucción entregada por el docente, por medio de una tarea específica o de un objetivo, como describir, explicar, fundamentar, etc., y, posteriormente, se analizó en cada caso la correspondencia entre lo que solicitaba el profesor, lo que respondía el estudiante y los recursos lingüísticos involucrados. A continuación, se separaron los textos en complejos clausales con el objeto de desarrollar la descripción de los pasos o etapas que los conformaban, y se registró esta información en una tabla. El análisis de cada pregunta-respuesta en las tablas especifica los pasos y fases del género, utilizando la notación de las relaciones de taxis entre cláusulas: parataxis (//) hipotaxis (/) cláusulas incrustadas ([ ]) inicio y término de complejo clausal $(/ / /)$. Además, se subrayaron los procesos y se utilizó negrita para destacar el elemento con el que se inicia cada fase identificada.

\section{Análisis y resultados}

\section{Las variables del registro en los textos estudiantiles}

El análisis de las variables del registro (tenor, campo y modo) en los textos que conforman el corpus de esta investigación muestra cómo se sitúa el discurso estudiantil en la tarea y los temas de las disciplinas abordadas.

En relación con el tenor, destaca el uso del lenguaje para la puesta en marcha de una relación entre evaluador (docente) y evaluado (estudiante). Dicha relación ofrece ciertas particularidades. Los estudiantes conocen, en general, el tipo de preguntas con las que serán evaluados y las preferencias del docente en relación con la extensión de las respuestas y la libertad para construir recontextualizaciones propias, pero, más allá de esto, advierten que se trata de un contexto diferente al cotidiano y familiar y que en él deben tratar de organizar sus respuestas de la mejor forma posible para obtener la calificación esperada.

Con respecto al campo, la experiencia que los estudiantes reconstruyen se refiere en forma estricta, con más o menos detalles, a los conocimientos disciplinares sobre los cuales son interpelados. El análisis de los ítemes léxicos permite especificar para cada área y nivel el campo del corpus en estudio, que coincide con las consignas de los profesores y que se sintetiza en el cuadro 2, aludiendo a los conceptos centrales (nombres) acerca de los cuales los estudiantes construyen sus textos. En Biología, los contenidos corresponden, en primer y tercer año de Enseñanza Media, 
a la unidad "Organismos, ambiente y sus interacciones". En Historia, los contenidos versan sobre "Historia universal" en primer año, e "Historia de Chile" en tercero.

\section{Cuadro 2.}

Síntesis del campo en el corpus en estudio

\begin{tabular}{cll}
\hline Área & \multicolumn{1}{c}{$\mathbf{1 .}^{\circledR}$ medio } & \multicolumn{1}{c}{ 3. medio $^{\circ}$} \\
\hline \multirow{2}{*}{ Biología } & Interacciones tróficas & Selección natural \\
& Tipos de pirámides tróficas & Tipos de selección natural \\
\hline \multirow{2}{*}{ Historia } & Evolución del mundo rural & $\begin{array}{l}\text { Acontecimientos durante } \\
\text { el régimen militar }\end{array}$ \\
\hline
\end{tabular}

Fuente: elaboración propia

En relación con el modo, el uso del canal escrito permite que los estudiantes razonen y organicen sus respuestas. En el aspecto formal, se aprecian diferencias entre las áreas, que tienen que ver con la cantidad de palabras que utilizan los estudiantes para construir sus respuestas. En Biología, las respuestas tienen una extensión mucho menor, como se aprecia en los ejemplos analizados. Otro aspecto del modo tiene que ver con que, al inicio, las respuestas frecuentemente retoman las palabras utilizadas en la pregunta como una forma de asegurar que se está contestando lo que se pide.

Ahora bien, en la intersección entre campo y modo se advierten consecuencias interesantes, ya que no es lo mismo escribir sobre un tema cotidiano de amplia difusión, que sobre conocimientos disciplinares que implican discursos verticales cuyos significados son menos dependientes del contexto y refieren a estructuras simbólicas especializadas de conocimiento explícito. Este es, justamente, el punto de partida de la reflexión de Bernstein (1999) acerca de las estructuras de conocimiento, que tienden a la horizontalidad en el caso de la Historia y a la jerarquización en el caso de las Ciencias. Esta diferencia que se plantea específicamente en el modo del registro, debe notarse también a nivel de género y lenguaje, y de ello se pretende dar cuenta a continuación.

\section{Los géneros pedagógicos en los textos estudiantiles}

El análisis comparativo de los textos pregunta-respuesta en contextos de evaluación escrita arroja la presencia de cinco tipos de géneros (véase el cuadro 3 ). 


\section{Cuadro 3.}

Géneros correspondientes a las preguntas-respuestas en los textos estudiantiles

\begin{tabular}{|c|c|c|c|}
\hline Área & Preguntas & $1 .^{\circ}$ medio & 3. ${ }^{\circ}$ medio \\
\hline \multirow{6}{*}{ Biología } & $\mathrm{Pl}$ & Explicación factorial & Explicación secuencial \\
\hline & p2 & Explicación factorial & Explicación secuencial \\
\hline & p3 & Reporte. Composición & Explicación factorial y secuencial \\
\hline & $\mathrm{p} 4$ & Explicación factorial & Explicación secuencial \\
\hline & p5 & Explicación secuencial & Reporte. Descripción \\
\hline & p6 & Explicación secuencial y factorial & Explicación secuencial \\
\hline \multirow{3}{*}{ Historia } & Pl & Relato histórico & Explicación factorial \\
\hline & p2 & - & Explicación consecuencial \\
\hline & P3 & - & Explicación consecuencial \\
\hline
\end{tabular}

Fuente: elaboración propia

A continuación, se presenta un ejemplo por cada nivel y área del análisis de los géneros pedagógicos encontrados en las pruebas examinadas y sus correspondientes fases o etapas constitutivas, además de una síntesis interpretativa sobre los géneros representados. En estos ejemplos es posible advertir la manera como los estudiantes se apropian del discurso de la Biología y de la Historia.

\section{Producción escrita de los estudiantes en el área de Biología}

En el primer nivel medio, frente a la pregunta (P5) la respuesta requiere identificar el fenómeno y explicarlo o, de manera más precisa, justificarlo. El género resultante corresponde a una explicación factorial, ya que se presenta un fenómeno y dos causas o factores, como se aprecia en el cuadro 4.

\section{Cuadro 4.}

Explicación factorial. Género pedagógico en Biología, $1^{\circ}$ medio

\begin{tabular}{|c|c|c|}
\hline Pasos del género & Fases & Texto (Bı-16) \\
\hline Fenómeno & Identificación & ///Es una pirámide de energía// \\
\hline Explicación & $\begin{array}{l}\text { Justificación } \\
1 \text { (causa) }\end{array}$ & $\begin{array}{l}\text { ya que se está midiendo la cantidad de energía } \\
\text { almacenada en cada nivel durante un periodo } \\
\text { determinado de tiempo, en este caso un año, // }\end{array}$ \\
\hline Fenómeno & Refuerzo & también nos damos cuenta //de que es de energía// \\
\hline Explicación & $\begin{array}{l}\text { Justificación } \\
2 \text { (causa) }\end{array}$ & $\begin{array}{l}\text { ya que las cantidades van disminuyendo//a } \\
\text { medida que va subiendo de nivel// y eso es } \\
\text { exactamente //lo que ocurre con la energía///. }\end{array}$ \\
\hline
\end{tabular}


La justificación se construye a partir de dos expansiones paratácticas de elaboración que expresan causalidad de manera interclausal y congruente a través de la conjunción "ya que". Ambas explicaciones aparecen introducidas por la identificación del fenómeno ("pirámide de energía"), que en el segundo caso es reforzada por medio del adverbio "también". La estructura del género que se muestra en las fases es recursiva, ya que se identifica el fenómeno, luego se expresa la primera causa, se refuerza la identificación y aparece el siguiente factor.

Si bien en las preguntas de este nivel y área hay un proceso intelectual de interpretación a partir de un estímulo, esta interpretación se dirige a la comprensión de la información entregada (hechos, datos) y no a la expresión de opiniones o juicios por parte de los estudiantes, lo que marcará una diferencia que debe tenerse en cuenta en relación con lo que ocurre en Historia.

En el tercer nivel medio, las preguntas tienen que ver con la comprensión de entidades y procesos relacionados con la selección natural. Los géneros que construyen los estudiantes son similares a los del nivel anterior - explicaciones factoriales y secuenciales y reporte- con la salvedad de que el reporte en tercero medio en lugar de centrarse en las partes de un todo (composición), se centra en la clasificación y descripción de un fenómeno (descripción).

En el cuadro 5 se muestra el análisis de la pregunta p4:

\section{Cuadro 5.}

Explicación secuencial. Género pedagógico en Biología, 3. medio

\begin{tabular}{|c|c|c|}
\hline Pasos del género & Fases & Texto (BıO-III7) \\
\hline \multirow{2}{*}{ Fenómeno } & Consecuencia 1 & $\begin{array}{l}\text { ///Se puede deducir /que los individuos no_ } \\
\text { presentaron ni una relación previa// y /que } \\
\text { pertenecen a distintos tipos de especies///. }\end{array}$ \\
\hline & Consecuencia 2 & $\begin{array}{l}\text { ///Lo más seguro es /que sus huellas se } \\
\text { hayan hecho en el mismo tiempo// o /de poco } \\
\text { intervalos de tiempo entre uno y otro///. }\end{array}$ \\
\hline \multirow{2}{*}{ Explicación } & $\begin{array}{l}\text { Justificación } \\
\text { l (Causa) }\end{array}$ & $\begin{array}{l}\text { ///Se puede demostrar esto// porque/ en el } \\
\text { momento en que se juntan// después no habría ni } \\
\text { descendencia mixta o con caracteres parecidos, } \\
\text { //lo que los divide en distintas especies//, }\end{array}$ \\
\hline & $\begin{array}{l}\text { Justificación } \\
2 \text { (causa) }\end{array}$ & $\begin{array}{l}\text { y ocurrió al mismo tiempo// porque una especie } \\
\text { quedó eliminada, demostrando selección } \\
\text { natural en el mismo intervalo de tiempo///. }\end{array}$ \\
\hline
\end{tabular}

Fuente: elaboración propia

La pregunta $\mathrm{P} 4$ guía el planteamiento de una hipótesis (consecuencias) acerca de la figura que sirve de estímulo. Aunque se plantea "qué se puede decir", el contexto (registro fósil, evolución de las especies, test de 
Biología) lleva a que el estudiante responda "se puede deducir", porque la deducción es parte del método científico. A pesar de las modalizaciones impersonales ("se puede") no se trata de interpretar, sino de demostrar; no se trata de argumentar, sino de experimentar o, en este caso, extraer conclusiones a partir de los datos del experimento. Así, por ejemplo, con las expresiones "se puede deducir" y "se puede demostrar" se marca el comienzo de la primera consecuencia y de la primera causa, en tanto que se utiliza "lo más seguro es" y "ocurrió [...] porque" para la segunda consecuencia y la segunda causa. En la construcción de las consecuencias, en este caso, predominan los procesos relacionales y mentales ("deducir", "presentaron", "pertenecen", "es", "hayan hecho"). En la construcción de las causas se destaca la presencia de expresiones de relación lógico-semántica congruente ("porque", "lo que"). Se trata, por tanto, de una explicación secuencial doble, pues cada efecto tiene su causa.

El cuadro 6 sintetiza los géneros que los estudiantes construyen para responder a las preguntas del área de Biología.

\section{Cuadro 6.}

Sintesis de géneros en las evaluaciones del área de Biología

\begin{tabular}{ll}
\hline \multicolumn{1}{c}{$\mathbf{1}^{\circ}$ medio } & \multicolumn{1}{c}{$\mathbf{3}^{\circ}$ medio } \\
\hline Reporte (composición) & Reporte (descripción) \\
\hline Explicación secuencial & \\
\hline Explicación factorial
\end{tabular}

Fuente: elaboración propia

El análisis muestra, por una parte, la presencia del reporte en ambos niveles. El reporte — también Ilamado informe (Moyano, 2013b)— es uno de los géneros más comunes usados para introducir a los alumnos en los hechos científicos y en la metodología de la investigación (Martin, 1993b), dado que se centra en entidades para describirlas, clasificarlas o describir las partes de un todo. Es, por tanto, un género indicativo de la comprensión y asimilación del conocimiento científico, que basa gran parte de su construcción en la observación. Siguiendo a Martin (1993b), el reporte, o más bien, el tratamiento con entidades de las ciencias —aun cuando dichas entidades estén vinculadas a procesos- es el género más importante en los libros de texto de ciencia y, por tanto, es de interés que sea manifestado explícitamente en situaciones de evaluación.

Por otra parte, las explicaciones secuenciales y factoriales, y con ello las relaciones lógicas de causalidad que dichas explicaciones conllevan y en las que se presentan secuencias de causas y efectos (secuenciales) 
o múltiples causas para un efecto (factoriales), son de relevancia en esta área, puesto que este tipo de relaciones se encuentran en la base de la experimentación y, en ciencias, las justificaciones y los argumentos están constituidos, precisamente, por los resultados y datos aportados por los experimentos. Por tanto, la presentación de gráficos y textos que informan acerca de experimentos sirven de estímulo a los estudiantes para construir respuestas que permitan determinar causas y efectos de un fenómeno. Observación y experimentación, así como la comunicación de las derivaciones de estas actividades a través del reporte y la explicación son nucleares para la construcción del discurso científico en los niveles de formación de los estudiantes.

\section{Producción escrita de los estudiantes en el área de Historia}

En el área de Historia, en el primer nivel medio, la P1 insta a los estudiantes a que "expliquen la evolución del mundo rural". El proceso relacional requerido (explicar) no necesariamente lleva a los estudiantes a producir textos donde las relaciones de causa-efecto sean prioritarias. De hecho, se trata de explicar la "evolución", es decir, los cambios sufridos en una cronología, por lo que las etapas en el tiempo serán las que organicen el discurso, como se observa en el ejemplo del cuadro 7.

\section{Cuadro 7.}

Relato histórico. Género pedagógico en Historia, 1. ${ }^{\circ}$ medio

\begin{tabular}{lll}
\hline $\begin{array}{c}\text { Pasos del } \\
\text { género }\end{array}$ & Fases & Texto (HC-I) \\
\hline
\end{tabular}

\begin{tabular}{|c|c|c|}
\hline Fondo & $\begin{array}{l}\text { Surgimiento } \\
\text { del mundo } \\
\text { rural }\end{array}$ & $\begin{array}{l}\text { ///El mundo rural surgió en el periodo llamado Neolítico///. } \\
\text { ///En ese momento de la historia se realizaban las } \\
\text { actividades de recolección y caza para poder sobrevivir///. } \\
\text { ///Al surgir la agricultura y la ganadería los humanos } \\
\text { dejaron de ser nómades// (se trasladaban de un lugar a } \\
\text { otro constantemente para poder recolectar y cazar)// y, } \\
\text { por lo tanto, pasaron a ser sedentarios///. ///Durante esta } \\
\text { época la agricultura era muy básica//, además, debido a } \\
\text { que luego de hacer el trabajo de cultivo y cuidado de los } \\
\text { animales, habían tiempos de ocio (tiempo libre) [en los que } \\
\text { surgieron otras actividades campestres (principalmente } \\
\text { "manualidades") para utilizar su tiempo en algo]///. }\end{array}$ \\
\hline $\begin{array}{l}\text { Registro } \\
\text { de etapas }\end{array}$ & $\begin{array}{l}\text { Tiempo } 1 \\
\text { (Edad Media) }\end{array}$ & $\begin{array}{l}\text { ///Durante la edad media la agricultura pudo progresar } \\
\text { un poco//, ya que surgen nuevas herramientas de } \\
\text { trabajo// (avanzó la tecnología) /como, por ejemplo, } \\
\text { los herrajes para los animales, / que facilitaban el } \\
\text { desarrollo de la actividad///. ///A pesar de los avances, } \\
\text { la agricultura seguía con un alto grado de precariedad, } \\
\text { //debido a que aún dependía enormemente de las } \\
\text { condiciones naturales y a su bajo rendimiento///. }\end{array}$ \\
\hline
\end{tabular}




\begin{tabular}{|c|c|c|}
\hline $\begin{array}{l}\text { Pasos del } \\
\text { género }\end{array}$ & Fases & Texto (HC-I) \\
\hline \multirow{3}{*}{$\begin{array}{l}\text { Registro } \\
\text { de etapas }\end{array}$} & $\begin{array}{l}\text { Tiempo } 2 \\
\text { (1492) }\end{array}$ & $\begin{array}{l}\text { ///En } 1492 \text { /(cuando se descubrió América)/ fueron } \\
\text { llevados animales y alimentos propios de América a } \\
\text { Europa, por ejemplo, el tomate, la papa, el maíz, etc./// } \\
\text { Esto cambió la agricultura, //ya que comenzaron a } \\
\text { utilizar productos provenientes de otros lugares///. }\end{array}$ \\
\hline & $\begin{array}{l}\text { tiempo } 3 \\
\text { (siglo xx) }\end{array}$ & $\begin{array}{l}\text { ///Entre los avances propios del siglo xx (en la Rev. } \\
\text { Industrial) surge el tractor///. ///Este gran avance } \\
\text { tecnológico realizaba dificiles tareas de manera rápida y } \\
\text { en escalas [que hasta el momento eran inimaginables]///. } \\
\text { ///Debido a estos avances mucha gente quedó } \\
\text { desempleada// y se vio en la obligación de migrar a otros } \\
\text { lugares (principalmente ciudades [que ofrecían trabajos } \\
\text { industriales])] en busca de trabajos///. ///Luego, además } \\
\text { de producir alimentos, producían madera, fertilizantes, } \\
\text { flores, plantas ornamentales, cuero, pieles, fibras, etc./// }\end{array}$ \\
\hline & $\begin{array}{l}\text { Tiempo } 4 \\
\text { (actualidad) }\end{array}$ & $\begin{array}{l}\text { ///Actualmente la agricultura depende en gran manera } \\
\text { de la tecnología, //ya que sin ella, la agricultura tendrá } \\
\text { un rendimiento bajísimo///. ///Existen distintos } \\
\text { cuidados y técnicas [que se emplean hoy en la } \\
\text { agricultura]//, los cuidados y especialidades requieren } \\
\text { de personas especializadas en el tema (principalmente } \\
\text { ingenieros agrarios)// y los técnicos han permitido } \\
\text { generar más ganancias y darle mayor rentabilidad a la } \\
\text { agricultura///.///La agricultura hoy se ha expandido } \\
\text { llegando incluso a otros continentes// (se } \underline{\text { da gracias }} \\
\text { al procesamiento, embalaje y mercado)///. }\end{array}$ \\
\hline
\end{tabular}

Fuente: elaboración propia

Las respuestas de los estudiantes están constituidas por textos que se organizan con una estructura principalmente de orden temporal (Antigüedad, Edad Media, época moderna, actualidad), lo que es característico del relato histórico, género propio de la crónica y que, junto a la explicación, el reporte y los procedimientos, conforman la familia de los informes. El foco de atención está puesto en la información y en la comprensión del proceso "evolutivo" experimentado por el mundo rural. El primer paso, también Ilamado "fondo", alude al origen de la agricultura, a las transformaciones sufridas por el mundo rural a lo largo de la historia y al paso de las sociedades nómades a sedentarias. A continuación, se marcan —por medio de grupos nominales y adverbios que expresan circunstancias de tiempo_ las diferentes épocas hasta el tiempo actual. Al Ilegar a la última etapa, algunos estudiantes realizan síntesis de lo expuesto, señalando las consecuencias de los avances tecnológicos y de los últimos cambios que ha experimentado la agricultura; esto implica que en Historia existe una tendencia —en ciernes en este nivel, ya que no todos los estudiantes lo hacen- a conferir un sentido o emitir juicios acerca de lo que se ha relatado. Ejemplos de estas síntesis se presentan a continuación: 
(1) "En resumen, el mundo rural antes concentraba gran parte de la población y debido a mejoras tecnológicas se redujo notablemente en poco tiempo". (HC-11)

(2) "El mundo rural ha demostrado un enorme avance con el paso de los años llegando a ser el ambiente natural irreemplazable y que debemos valorar y cuidar". (HC-I2)

(3) "En conclusión podemos decir que la agricultura ha experimentado cambios gracias a los avances tecnológicos que el ser humano ha ido descubriendo, que han provocado consecuencias buenas y malas, una mala ha sido que la gente ha perdido la relación familiar que se tenía en el campo sin las máquinas". (HC-13)

Los estudiantes eligen finalizar sus respuestas con un comentario que contrasta el pasado (antes) con el presente (ejemplo 1), valorando positivamente el avance del mundo rural (ejemplo 2), o juzgando las consecuencias que la tecnología ha tenido en este (ejemplo 3 ).

En el tercer nivel medio, una de las preguntas (P1) demanda que se "explique por qué se plantea que a partir del gobierno militar se habría suprimido el Estado de Derecho". Esta pregunta, antes que explorar las causas por las que el Gobierno habría suprimido el Estado de derecho — dejando en suspenso el juicio acerca de lo sucedido-, busca evidenciar los hechos (consecuencias) que fundamentan tal observación. Frente a esta pregunta, los estudiantes contestan rescatando, en algunos casos, la fórmula impersonal que aparece en la pregunta ("se plantea que...") y pasando por alto el uso modalizado ("se habría suprimido") que se hace en la consigna acerca de la situación sobre la cual se pregunta. Los estudiantes responden acerca de "la supresión" o simplemente dicen que "se suprime", "se suprimió" o "fue suprimido" el Estado de Derecho.

Más adelante, los estudiantes emplean conectores causales para explicar en qué consistió dicha supresión. Las respuestas a esta pregunta corresponden a una explicación consecuencial, dado que se alude a múltiples efectos (fenómenos) para una casusa (factor), como se refleja en el cuadro 8.

\section{Cuadro 8.}

Explicación consecuencial. Género pedagógico en Historia, 3. medio

\begin{tabular}{ccc}
\hline $\begin{array}{c}\text { Pasos del } \\
\text { género }\end{array}$ & Fases & Texto (HC-III2) \\
\hline
\end{tabular}




\begin{tabular}{|c|c|c|}
\hline $\begin{array}{l}\text { Pasos del } \\
\text { género }\end{array}$ & Fases & Texto (HC-III2) \\
\hline \multirow{7}{*}{ Factores } & Efecto 1 & disolviendo el congreso de la época, \\
\hline & Efecto 2 & eliminando a todos los partidos correspondientes a la UP//. \\
\hline & Efecto 3 & $\begin{array}{l}\text { //También está la supresión de la central única } \\
\text { de trabajadores (cuT)//. Suprimiendo de esta } \\
\text { manera toda institución democrática//. }\end{array}$ \\
\hline & Efecto 4 & $\begin{array}{l}\text { //Sin mencionar /que se suprimieron } \\
\text { las elecciones y el voto//. }\end{array}$ \\
\hline & Efecto 5 & $\begin{array}{l}\text { //También se produjo una gran represión por parte del } \\
\text { estado de sitio, la ley marcial y el toque de queda// }\end{array}$ \\
\hline & Efecto 6 & $\begin{array}{l}\text { lo que causó una supresión a las manifestaciones y a las } \\
\text { reuniones o juntas clandestinas de la oposición u otro partido }\end{array}$ \\
\hline & Efecto 7 & $\begin{array}{l}\text { '// lo que causó un receso en todas las } \\
\text { actividades de distintos partidos políticos no } \\
\text { pertenecientes al Régimen militar///. }\end{array}$ \\
\hline Reafirmación & $\begin{array}{l}\text { Síntesis y } \\
\text { comentario }\end{array}$ & $\begin{array}{l}\text { ///Gracias a todo esto se suprimió el estado de derecho } \\
\text { en las instituciones y en las personas de manera } \\
\text { individual// ya que el miedo te obligaba a pensar de } \\
\text { solo una forma y a no manifestarte, [(algo que es) } \\
\text { un derecho básico dentro de cada persona]///. }\end{array}$ \\
\hline
\end{tabular}

Fuente: elaboración propia

En este ejemplo, el estudiante recurre a una variedad de expresiones, tales como relaciones lógico-causales congruentes ("ya que") y no congruentes ("lo que causó"), formas no finitas ("disolviendo", "eliminando", "suprimiendo"), conjunciones copulativas ("también") y otras expresiones retóricas ("sin mencionar") para explicar por qué se plantea que se habría suprimido el Estado de derecho. La explicación se cierra con una síntesis y comentario, introducida por la conjunción causal "gracias a", como si se tratara de un hecho positivo, contraponiéndose con "el miedo" y la obligación "a pensar de solo una forma y a no manifestarte", que esta situación había provocado.

El cuadro 9 sintetiza los géneros encontrados en los textos producidos en el área de Historia en situación de evaluación en los diferentes niveles.

\section{Cuadro 9.}

Sintesis de géneros en las evaluaciones del área de Historia

\begin{tabular}{lc}
\hline \multicolumn{1}{c}{$\mathbf{1}^{\circ}$ medio } & \multicolumn{3}{c}{$\mathbf{3}^{\circ}$ medio } \\
\hline Relato histórico & Explicación factorial y consecuencial \\
\hline Foco: la información (comprender procesos históricos) & \\
\hline
\end{tabular}


Se observa que los géneros pedagógicos de la historia se concentran en la comprensión de procesos y tienen como propósito primordial informar al lector acerca de esta comprensión de los hechos aludidos. Las preguntas de estos niveles se centran en el qué (la evolución del mundo rural, la doctrina de seguridad nacional, el proyecto de refundación política del régimen militar) y en el porqué (la supresión del Estado de derecho). De esta manera, el profesor está evaluando el manejo que los estudiantes tienen de la información entregada, aunque no por ello queda absolutamente fuera el carácter interpretativo de los hechos relatados o explicados. De manera casi indisociable, y quizás desde niveles anteriores a primero medio, los estudiantes no solo exponen los hechos sino también dejan entrever alguna valoración sobre estos. En efecto, como señala Moyano, un aspecto relevante en el discurso de la historia es la construcción de la valoración, que "implica no solo la evaluación de hechos y participantes, sino también la construcción de acuerdos y desacuerdos con otros hablantes e, incluso, con los lectores"(2013a, p. 68).

\section{Conclusiones}

La caracterización realizada de los géneros presentes en los textos producidos por estudiantes en el registro de las áreas de Biología e Historia en contextos de evaluación escrita coincide con las características del discurso vertical de las ciencias y las humanidades y con los géneros pedagógicos identificados en el marco de las investigaciones en las escuelas australianas. De acuerdo con los géneros empleados en este nivel, tanto en Biología como en Historia el foco de interés está puesto en la comprensión, de entidades y procesos, en el primer caso, y de procesos, en el segundo.

En Biología, el género del reporte está presente en ambos niveles, al igual que las explicaciones factoriales y secuenciales. Se trata, justamente, de dos familias de géneros característicos del discurso de la ciencia (Martin y Rose, 2008). Según Martin (1993b), la clasificación y la composición, que forman parte del reporte, cumplen un rol significativo en los textos científicos, ya que hacen posible destacar términos y definiciones, a la vez que producir descripciones de un fenómeno, de tipos de fenómenos o de las partes de un todo. Con respecto a las explicaciones secuenciales y factoriales, estas permiten el desarrollo del pensamiento científico, al establecer las conexiones causales, basadas en datos y resultados de experimentos, que son necesarias para explicar un fenómeno.

En Historia, se utilizan dos géneros del informe que se centran en la comprensión de procesos. Se trata de un género propio de la historia: el relato histórico, que marca las etapas en la evolución del mundo rural, y de las explicaciones factoriales, secuenciales y consecuenciales que 
remiten a una multiplicidad de factores o de efectos en las relaciones causales que manifiestan y, por tanto, dan cuenta de la complejidad de los procesos sociales. En los textos estudiantiles de esta área, la comprensión de los procesos históricos está matizada por valoraciones y juicios que indican, por una parte, la adopción de una perspectiva o una posición ideológica por parte de quien escribe y, por otra, la fuerte tendencia a "interpretar", a conferir un sentido o atribuir una intención.

Las diferencias en los géneros utilizados por estudiantes en formación en las áreas investigadas y en la conformación de estos reflejan de manera incipiente las distinciones entre estructuras del conocimiento científico y humanístico. En biología, se destacan la observación y la inferencia lógica basadas en la experimentación. En historia, la expresión de juicios y puntos de vista acerca de los sucesos, pues muchos de los relatos y explicaciones terminan con una apreciación acerca de lo expuesto. La importancia de asumir una postura con respecto a los eventos históricos reseñados resulta patente en la escritura estudiantil.

De los análisis realizados desde la perspectiva del género se desprende que aun cuando los profesores no enseñen de manera consciente a sus estudiantes las características propias del discurso vertical, el hecho de que evalúen a sus estudiantes a través de géneros de los discursos científicos y humanistas es un indicador, para estos últimos, de ciertas diferencias y rasgos de la disciplina en la que se están formando. La construcción del conocimiento con una estructura vertical (como en las ciencias) u horizontal (como en las humanidades) no es un asunto ignorado por los estudiantes, pues en su formación se les induce a uno u otro tipo de recontextualización del conocimiento.

\section{Referencias}

Bernstein, B. (1999). Vertical and horizontal discourse: An essay. British Journal of Sociology of Education, 20(2), 157-173.

Christie, F. (1998). Learning the literacies of primary and secondary schooling. En F. Christie y R. Misson, Literacy and schooling (pp. 47-73). Routledge.

Christie, F. (2012). Language education throughout the shool years: A functional perspective. Wiley-Blackwell.

Coffin, C. (2000). Constructing and giving value to the past: An investigation into secondary school history. En F. Christie y J. Martin, Genre and institutions: Social processes in the workplace and school (pp. 196-230). Continuum.

Eggins, S. (1994). An introduction to systemic functional linguistic. Cassell. 
Eggins, S. y Martin, J. R. (2003). El contexto como género: Una perspectiva lingüística funcional. Revista Signos, 36(54), 185-205.

Eggins, S., Wignell, P. y Martin, J. (1993). The discourse of history: Distancing the recoverable past. En M. Ghadessy, Register analysis: Theory and practice (pp. 75-109). Pinter Publishing.

Halliday, M. (1993). Some grammatical problems in scientific English. En M. Halliday y J. Martin, Writing science: Literacy and discursive power (pp. 76-94). The Falmer Press.

Halliday, M. y Hasan, R. (1989). Language, context and text: Aspects of language in a social semiotic perspective. Oxford University Press.

Halliday, M. y Martin, J. (1993). Writing science: Literacy and discursive power. The Falmer Press.

Martin, J. (1985). Process and text: Two aspects of semiosis. En J. Benson y W. Greaves, Systemic perspectives on discourse (Vol. 1: Selected theoretical papers from the 9th International Systemic Workshop, pp. 248-74). Ablex.

Martin, J. (1992). English text: System and structure. John Benjamins.

Martin, J. (1993a). Life as a noun: Arresting the universe in sciences and humanities. En M. Halliday y J. Martin, Writing science: Literacy and discursive power (pp. 242-293). The Falmer Press.

Martin, J. (1993b). Literacy in science: Learning to handle text and technology. En M. Halliday y J. Martin, Writing science: Literacy and discursive power (pp. 242-293). The Falmer Press.

Martin, J. (2000). Analysing genre: Functional parameters. En F. Christie y J. Martin, Genre and institutions: Social processes in the workplace and school (pp. 3-39). Continuum.

Martin, J. (2014). Evolving systemic functional linguistics. Functional Linguistics, 1(3).

Martin, J., Eggins, S., Wignell, P. y Rothery, J. (1988). Secret English: Discourse technology in the junior secondary school. En L. Gerot, J. Oldenburg y T. Van Leeuwwen, Language and socialisation, home and school: Proceedings from the Working Conference on Language in Education. Macquarie University.

Martin, J. y Rose, D. (2008). Genre relations: Mapping culture. Equinox Publishing.

Martin, J. y Rothery, J. (1993). Grammar: Making meaning in writing. En B. Cope y M. Kalantzis, The powers of literacy: A genre approach to teaching writing (pp. 137-153). The Falmer Press.

Morris, J. P. y Navarro, F. (2011). Registro y género: Cómo entiende la lingüística sistémico funcional las clases estables de textos. Texturas, 10(11), 67-85. 
Moyano, E. (2007). Enseñanza de habilidades discursivas en español en contexto pre-universitario: Una aproximación desde la LSF. Signos 40(65), 573-608. http://dx.doi.org/10.4067/S0718-09342007000300009

Moyano, E. (2011). Deconstrucción y edición conjuntas en la enseñanza de la escritura: La reflexión sobre género y discurso en la formación académica y profesional. Anais vi Simposio Internacional de Estudios de Generos Textuais. Universidade Federal do Rio Grande do Norte.

Moyano, E. (2013a). El lenguaje de las disciplinas y los géneros de su recontextualización escolar. Una aproximación desde la lingüística sistémico-funcional. En E. Moyano, Aprender Ciencias y Humanidades: una cuestión de lectura y escritura (pp. 31-78). Universidad Nacional de General Sarmiento.

Moyano, E. (2013b). Géneros y discurso en los manuales de Biología: construcción del conocimiento y la actividad científica. En E. Moyano, Aprender ciencias y humanidades: una cuestión de lectura y escritura (pp. 229-296). Universidad Nacional de General Sarmiento.

Rose, D. y Martin, J. (2012). Learning to write: Reading to learn. Equinox.

Veel, R. (2000). Learning how to mean-scientifically speaking: Apprenticeship into scientific discourse in the secondary school. En F. Christie y J. Martin, Genre and institutions: Social processes in the workplace and school (pp. 161-195). Continuum.

Wignell, P. (2007). On the discourse of social science. Charles Darwin University Press. 